\title{
Wideband Radio Propagation Modeling for Indoor Geolocation Applications
}

\author{
Kaveh Pahlavan, Prashant Krishnamurthy, and Jacques Beneat \\ Worcester Polytechnic Institute
}

\begin{abstract}
A framework for statistical modeling of the wideband characteristics of the frequency-selective fading multipath indoor radio channel for geolocation applications is presented. Multipath characteristics of the channel are divided into three classes according to availability and the strength of the direct line of sight (DLOS) path with respect to the other paths. Statistics of the error in estimating the time of arrival of the DLOS path in a building is related to the receiver's sensitivity and dynamic range. The effects of external walls on estimating the location of the DLOS path are analyzed.
\end{abstract}

need to identify the location of portable and in-demand pieces of equipment. Public safety departments are thinking of identifying the location of people at the site of a crime or accindoor radio channels suffer from extremely serious multipath conditions that have to be modeled and analyzed to enable the design of radio equipment for a variety of applications. The objective of wideband radio propagation modeling for telecommunications and geolocation applications are quite different. As a result, available models for radio channel propagation are not adequate for analyzing the performance of geolocation systems. In radio propagation studies for telecommunication applications, the main objective is to determine the relationship between distance and total received power in all paths, and to find out the multipath delay spread of the channel. The distancepower relationship is used to determine the coverage of the radio and the multipath delay spread to evaluate the data rate limitations of the receivers [1]. The objective of radio propagation studies for geolocation applications is to determine the relative power and time of arrival (TOA) of the signal arriving from the direct line of sight (DLOS) path versus the signal arriving from other paths. The relative power and TOA of the paths, and the channel noise and interference are used to analyze the error in estimating the distance between the transmitter and the receiver if the DLOS path is not detected correctly.

With the increased popularity of wireless services in the 1990 s new applications in a variety of fields have evolved. These applications were incentives for radio propagation measurement and modeling in indoor and outdoor areas. In the telecommunications industry, indoor radio propagation studies were motivated by voice-oriented wireless private branch exchange (PBX) and personal communications services (PCS) applications as well as data-oriented wireless LANs and wireless ATM services [2]. Wideband radio propagation studies were more focused on wideband data applications such as LAN extension, inter-LAN bridges, nomadic access, ad hoc networking, and fusion of computers and communications [3]. Research in wideband indoor radio channel modeling for telecommunications applications in the past decade resulted in numerous measurements, statistical models, and ray tracing software to identify the wideband characteristics of different classes of buildings such as factory floors, office buildings, and residential houses [1].

More recently, applications for indoor geolocation are becoming popular [4]. In mental hospitals and jails there is a growing need to identify the location of specific patients or inmates. In warehouses, laboratories, and hospitals there is a

dent [5-7]. Fire department officers are keen on identifying the locations of victims of accidents and firefighters inside a building. Small unit operation (SUO) military teams are keen on situation awareness systems (SASs) capable of identifying the location of individual warfighter systems (IWSs) inside buildings [8]. Quantitative study of the feasibility of alternative indoor geolocation systems for these applications requires measurement and modeling of the indoor radio channel to predict and analyze the availability of the DLOS path in different parts of a building. In the same way that the bit error rate (BER) is the ultimate measure for comparing performance of different digital communication receivers, accuracy of measurement of the TOA of the DLOS path is a measure of the performance of geolocation receivers. In this article we provide a new framework to model the radio propagation characteristics for analysis of the TOA of the DLOS path.

\section{THREe Classes of Locations}

In wideband indoor radio propagation studies for telecommunication applications often channel profiles measured in different locations of a building are divided into line of sight and obstructed line of sight because the behavior of the channel in these two classes has substantially different impacts on the performance of a telecommunications system. A logical way to classify channel profiles for geolocation applications is to divide them into three categories. The first category is the dominant direct path (DDP) case, in which the DLOS path is detected by the measurement system and is the strongest path in the channel profile. In this case, traditional GPS (Global Positioning System) receivers [9-11] can lock onto the DLOS path and detect its TOA accurately. The second category is the nondominant direct path (NDDP) case, where the DLOS path is detected by the measurement system but is not the dominant path in the channel profile. For these profiles traditional GPS receivers, expected to lock onto the strongest path, will make an erroneous decision on the TOA. The amount of error made by a traditional receiver is the distance associated with the difference between the TOA of the strongest path and the TOA of the DLOS path. For the second category, locations with NDDP profiles, a more complex RAKE type receiver [1] can resolve the multipath and make an intelligent decision on the TOA of the DLOS path. The third category of channel profiles are unde- 


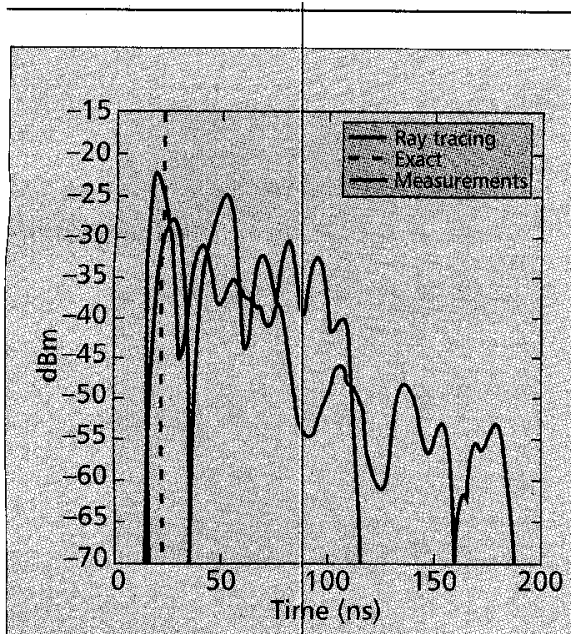

(a)

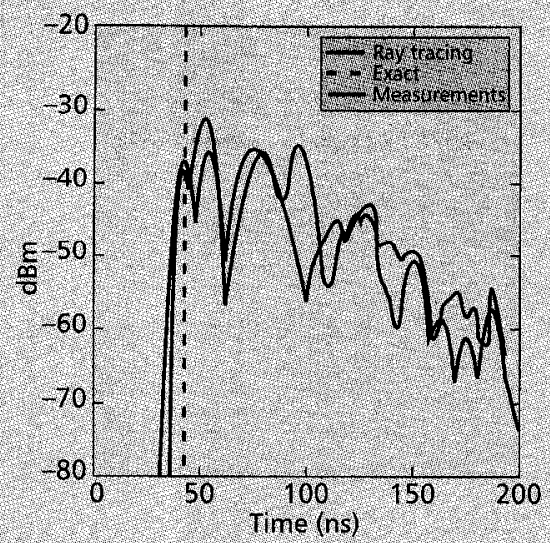

(b)

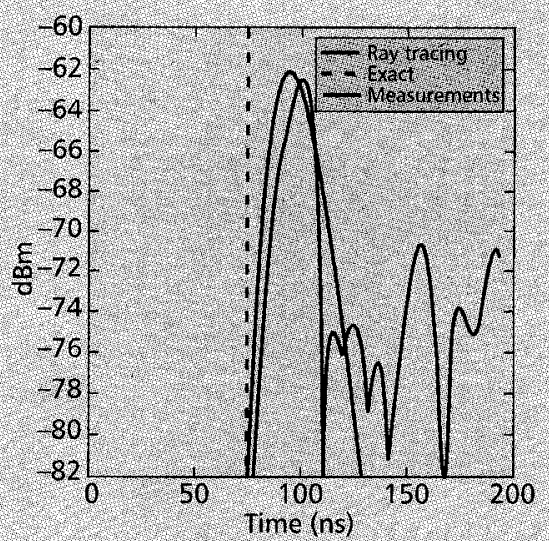

(c)

Figure 1. Three classes of channel profiles: a) DDP, b) NDDP, and c) UDP.

tected direct path (UDP) profiles. In these profiles the measurement system cannot detect the DLOS path, and therefore neither traditional GPS nor RAKE-type receivers can detect the DLOS path. If we define the ratio of the power of the strongest path to the power of the weakest detectable path of a profile as the dynamic range of a receiver, in NDDP profiles the strength of the DLOS path is within the dynamic range of the receiver, and in UDP profiles it is not. If practical considerations regarding the dynamic range are neglected, one can argue that we have only two classes (DDP and NDDP) of profiles because the DLOS path always exists, but sometimes we cannot detect it with a practical system.

\section{DESCRIPTION OF THE MEASUREMENTS}

The measurement system we have used is the frequency domain measurement system described in [12]. The centerpiece of this system is a network analyzer that sweeps the channel from 900-1 $100 \mathrm{MHz}$. The output signal is first amplified with a $27 \mathrm{dBm}$ amplifier and then connected to the transmitter antenna by a long cable. The receiver antenna passes the signal through a chain of low-noise amplifiers that are connected to the input port of the network analyzer. The network analyzer records the frequency response of the channel and, by taking the Fourier transform, provides the impulse response we refer to as a channel time profile or simply profile. The sensitivity of the network analyzer is $-\$ 0 \mathrm{dBm}$. Running massive simulations requires exhaustive results from the measurement system, which is extremely expensive. To avoid this difficulty, we use the CWINS ray tracing. (RT) software described in [13-15] to draw our statistical conclusions. Recently, RT has also been used for development of wideband statistical models for indoor telecommunications applications [15]. In general, all the details of building and furniture as well as movement of people are not included in any $\mathrm{RT}$ programs. We increase details of the building to the extent that results of empirical data from the measurement system at selected points agree with results of RT for the particular application. From that point onward, we use results of RT for massive simulations to draw reasonable statistical conclusions $[1,14,16]$. For telecommunications applications we are interested in the total received power and root mean square (rms) multipath delay spread of the channel. For geolocation applications we have included adequate details of the building to match the power of the DLOS path and to an extent the power in the remaining paths.

Figure 1 shows samples for the three classes of profiles obtained from RT and the measurement system on the first floor of the Atwater Kent (AK) Laboratories at Worcester Polytechnic Institute. The floor plan of this building and the location of the transmitter and receivers are shown in Fig. 2.

In the DDP case the transmitter and receiver are in the same area, in the NDDP case a couple of walls separate them, and in the UDP case several walls are between them. As shown in Fig. 1, results of measurement and RT show close agreement in the DDP and NDDP cases. The DLOS path is within 2-4 dB, the range of variations of the paths is within a few $\mathrm{dB}$, and the arriving paths from $\mathrm{RT}$ have a reasonable match to the result of measurements. For the UDP case RT accurately predicts the lack of the DLOS path, and the dynamic range is within a few $\mathrm{dB}$, but the rest of the paths follow less accurately compared to the other two cases. As we mentioned earlier, these differences are caused by effects of movement and other details not included in the RT program. However, in geolocation applications we are mainly concerned with statistical bèhavior of the DLOS path and the dynamic range of the signal. For these purposes RT proves to be a reliable tool for modeling.

\section{StATISTICAL BEHAVIOR OF THE ChANNEL}

To investigate the statistical behavior of the strength of the DLOS path, RT simulations were performed for 1600 receiver locations in a grid covering the first floor of the AK Laboratories, the floor plan of which is shown in 


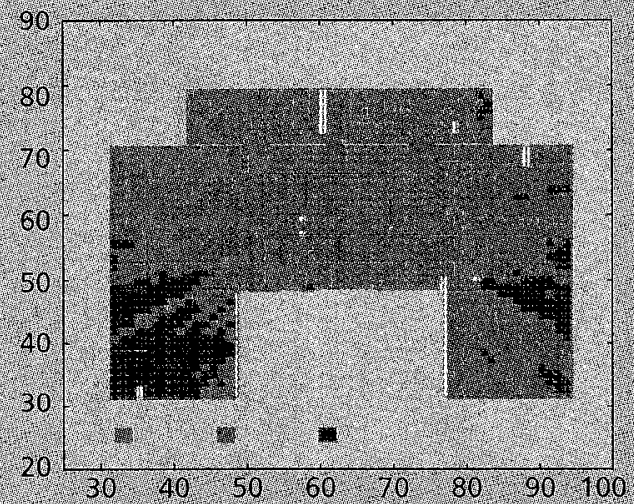

Figure 3. Locations in the building exhibiting the three classes of multipath profiles for a receiver sensitivity of $-80 \mathrm{dBm}$ and $40 \mathrm{~dB}$ dynamic range.

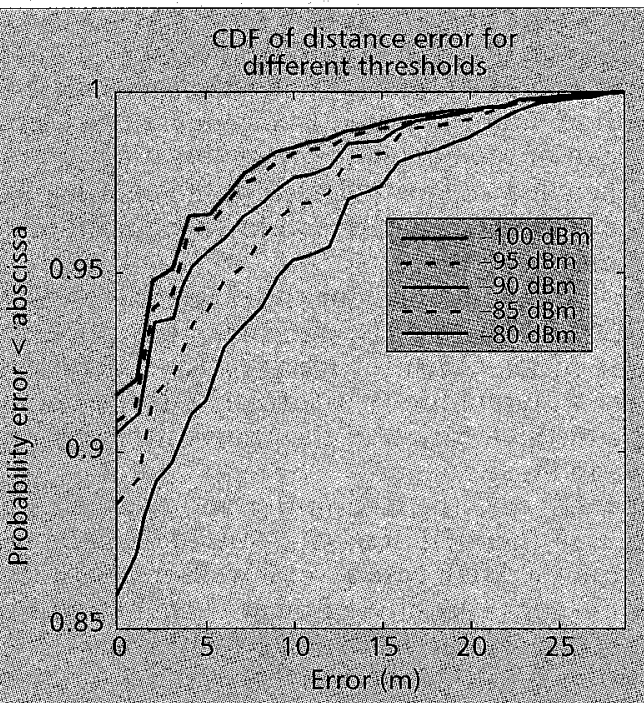

(a)

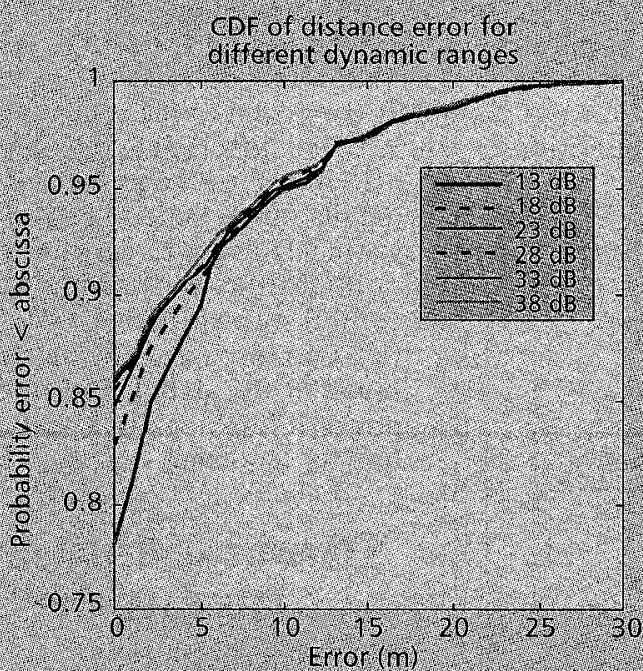

(1).

I Figure 5. $C D F$ of the error in predicted distance for the indoor transmitter: a) as a function of different receiver sensitivities and a fixed dynamic range of $40 \mathrm{~dB} ; b$ ) as a function of different receiver dynamic ranges and a fixed sensitivity of $-80 \mathrm{dBm}$.

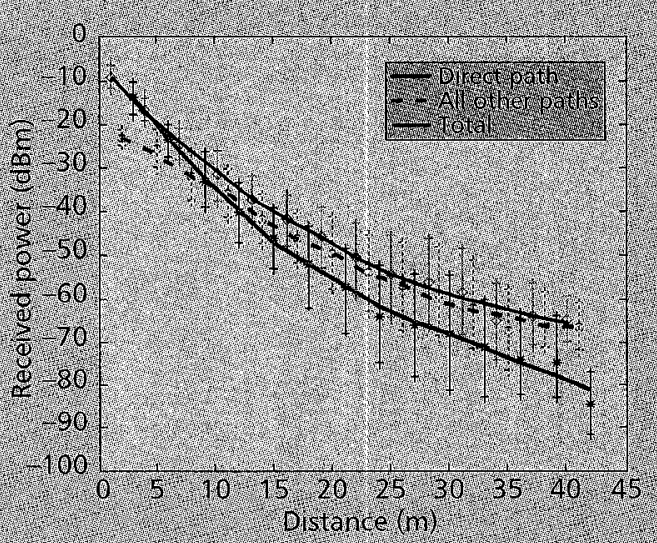

Figure 4. Received power as a function of distance in the $A K$ Laboratories first floor for the indoor transmitter location shown in Fig. 2.

Fig. 2. The transmitter is located at the center of the building, and the receiver is moved to different points on the grid. The AK building was built in 1906 and had two major remodelings and additions in 1934 and 1981. Therefore, in some areas within the building we have more than one exterior-type wall. The exterior walls of this building are heavy brick, the interior walls are made of aluminum stud and sheet rock, the floors are made with metallic beams, the doors and windows are metallic, and many other metallic objects (such as relatively large electric motors, equipment, and vending machines) are spread over different laboratory areas and corridors of the first floor. The excessive number of metallic objects and heavy and multiple external walls makes this building a very harsh environment for radio propagation.

Figure 3 shows the classes of channel profiles obtained in different locations of the building. The red, green, and blue areas correspond to the DDP, NDDP, and UDP profiles, respectively. As we discussed earlier, in the red areas traditional geolocation systems work properly, in the green areas more complicated RAKE type receivers are needed to accurately extract the TOA of the DLOS path, and in the blue areas we need additional transmitters to measure the distance accurately. The reader should be reminded that in practice a geolocation system involves at least three transmitters spread over opportunistically selected locations in the building. This figure provides an intuitive understanding of the range of operation of one reference transmitter operating inside a harsh indoor environment.

We next examine the strength of the DLOS path compared to the combined strength of all other paths and the total received power, including the power in the DLOS path. Figure 4 shows the received power (in $\mathrm{dBm}$ ) versus distance for the DLOS path, all other paths combined, and total power for all locations of the floor plan. The vertical lines on the curves correspond to the one standard deviation of the received signal strength for that distance.

Over a distance of $45 \mathrm{~m}$ in this building, the range of received power in the DLOS path is around $75 \mathrm{~dB}$, while the range of received power in combined other paths and total received power is around $40 \mathrm{~dB}$. The range of received power in the DLOS path is noticeably larger than that of the other paths combined and total received power. An immediate conclusion from this observation is that the receivers designed for geolocation applications in a frequency-selective fading multipath environment should accommodate wider dynamic ranges for the received signal power. 


\section{EFFECTS OF RECEIVER DESIGN}

The main objective of a geolocation radio receiver is to detect the TOA of the DLOS path. The DLOS path always exists, but in practice for a given receiver sensitivity, dynamic range, and bandwidth, we cannot correctly measure the TOA of the DLOS path all the time. Assuming that the bandwidth is sufficiently large to resolve all the paths, if a receiver cannot detect the DLOS path it will assume that the first detected path is the DLOS path, and will make an erroneous decision on estimating the TOA. This error can easily be mapped to the error in measuring the distance between the transmitter and receiver. To further analyze the behavior of the channel for geolocation applications we look at the effects of sensitivity and dynamic range of a wideband receiver on the probability of detection of the DLOS path and the cumulative distribution function (CDF) of the error in measurement of the distance between the transmitter and receiver.

Detection of the TOA is a function of multipath structure, receiver specification, signal-to-noise ratio, and the algorithm used to detect the DLOS path. We assume a wideband RAKE-type receiver capable of resolving the entire multipath profile of the channel. This receiver declares the TOA of the first detected path as the TOA of the DLOS path. We identify this receiver by its sensitivity and dynamic range. Sensitivity provides the minimum power level of a path that can be detected. Dynamic range provides the difference in $\mathrm{dB}$ between the strongest and weakest detectable paths. For example, when assuming a sensitivity of $-80 \mathrm{dBm}$ and a dynamic range of $15 \mathrm{~dB}$, the DLOS path is detectable if its power is stronger than $-80 \mathrm{dBm}$ and is not more than $15 \mathrm{~dB}$ below the power of the strongest path. If these two conditions are not satisfied, the receiver detects the first available path that fits this condition. When the receiver detects another path the difference between the distances measured based on the TOA of the erroneously detected path and the TOA of the DLOS path is considered as the error in detection.

Figure 5a shows the CDF of the distance error as a function of receiver sensitivity for a fixed dynamic range of 40 $\mathrm{dB}$, using the same locations described in Fig. 3. A change in receiver sensitivity from $-80 \mathrm{dBm}$ to $-100 \mathrm{dBm}$ will change the probability of correct detection of the DLOS path from 86 to 92 percent. The majority of errors occur for shorter distances, for example, a receiver with $-100 \mathrm{dBm}$ sensitivity makes an error of less than $2 \mathrm{~m} 95$ percent of the time, although, with a low probability, the receiver may make errors up to $30 \mathrm{~m}$. The larger errors belong to cases where several walls separate the transmitter and receiver. In these cases, often the overall received signal is extremely weak and all paths arrive after several reflections and transmissions through the walls. Again, the reader should note that the errors in Fig. 5 are the errors of a single receiver and not the error of the geolocation system. Practical geolocation systems usually provide redundancies by installing additional reference transmitters and use intelligent algorithms to avoid erroneously reported distances [11]. As a result, the system error can be reduced so that it is significantly less than the error of each individual receiver. When the overall architecture of the geolocation system is specified, statistics provided in Fig. 5 can be used to analyze and determine the actual error of the system.

In the implementation of a receiver, the dynamic range is related to the sidelobes of the pulses that are used for measurement of the TOA. For example, if Nyquist pulses are used, the sidelobes are about $13 \mathrm{~dB}$ below the main

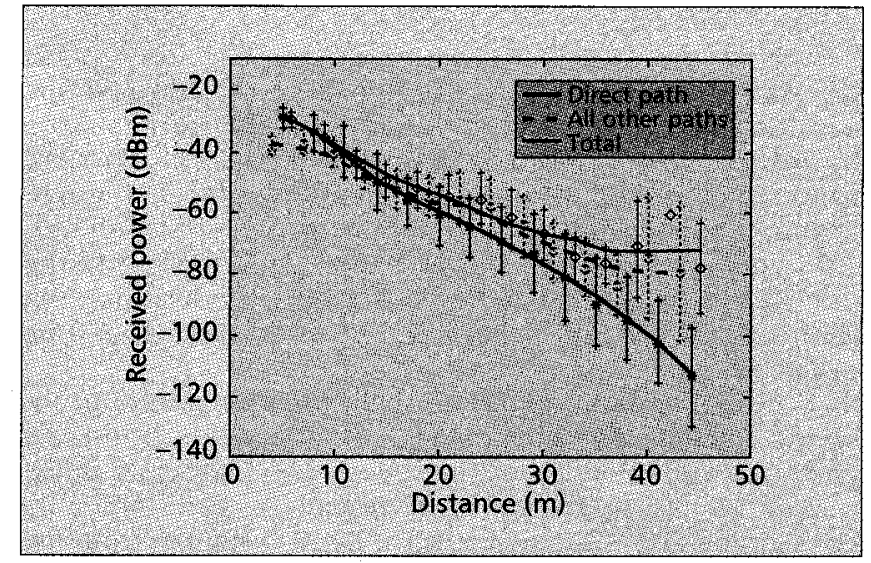

Figure 6. Received power as a function of distance in AK Laboratories first floor for the outdoor transmitter location shown in Fig. 2.

peak. Therefore, if a path is more than $13 \mathrm{~dB}$ below the strongest path it cannot be detected, and the dynamic range of this receiver is $13 \mathrm{~dB}$. Using Hanning or other low-sidelobe pulses, we can increase the dynamic range to more than $40 \mathrm{~dB}$. Figure $5 \mathrm{~b}$ shows the effects of the receiver dynamic range on the CDF of the distance error in meters for a receiver with a sensitivity of $-80 \mathrm{dBm}$. A change in dynamic range from $38 \mathrm{~dB}$ to $13 \mathrm{~dB}$ will reduce the probability of detection of the DLOS path from 86 to 78 percent. For a dynamic range of $13 \mathrm{~dB}$, in more than 90 percent of locations the error in measuring the distance is less than $5 \mathrm{~m}$. Analysis of this sort is useful for receiver designers to evaluate the trade-off among different pulse shapes and relate them to the overall performance of the geolocation system.

\section{EFFECTS OF EXTERNAL WALLS}

So far in this article, we have analyzed characteristics of the radio channel for indoor-to-indoor applications, such as geolocation systems for hospitals or manufacturing floors, where the reference transmitters are installed inside the building. There are other indoor applications for which the reference transmitters must be located outside the building. For example, in a military or firefighting operation a warfighter or firefighter could be inside the building while the rest of the troops are outside in nearby locations around the building. In these situations it is expected that the reference transmitters will be located outside the building near the external walls while the receiver is inside the building.

When we move the transmitter outside the building, characteristics of radio propagation change significantly [1]. This abrupt change of propagation characteristics is caused due to two major reasons: excessive indoor penetration loss through external walls of the building, and additional paths arriving through windows and doors reflected from neighboring buildings. External walls are usually thicker loadbearing walls made of heavier material which may include concrete and metallic beams. The outside of these walls are covered by external siding that is sometimes metallic, and the insides of these walls are filled with insulation material. As a result, in- building penetration loss of external walls is 10-15 $\mathrm{dB}$ higher than the loss caused by passing through traditional internal walls [1]. When the transmitter is outside the building, because of this extra power loss due to in-building penetration, the paths that arrive after penetration though several walls become significantly weak and, at 
certain points, weaker than the signals coming through the windows and doors after bouncing off the neighboring buildings. When both the transmitter and receiver are inside the building we usually neglect the effects of neighboring buildings and the signal penetrations through outside windows and doors because the signal must cross the outside walls twice to come back inside the building. At that stage the signal is assumed to be so weak it can be neglected.

For telecommunication applications the effects of inbuilding penetration phenomena are reflected in additional path loss and an increase in average rms multipath delay spread. To observe the effects of in-building penetration on geolocation applications, we move the transmitter from the center of the building to the outside location shown in Fig. 2 and repeat our previous experiments and analysis. Figure 6 shows the received power versus distance in meters and the best fit curve for the DLOS path, combined other paths, and total received power. The range of the received signal

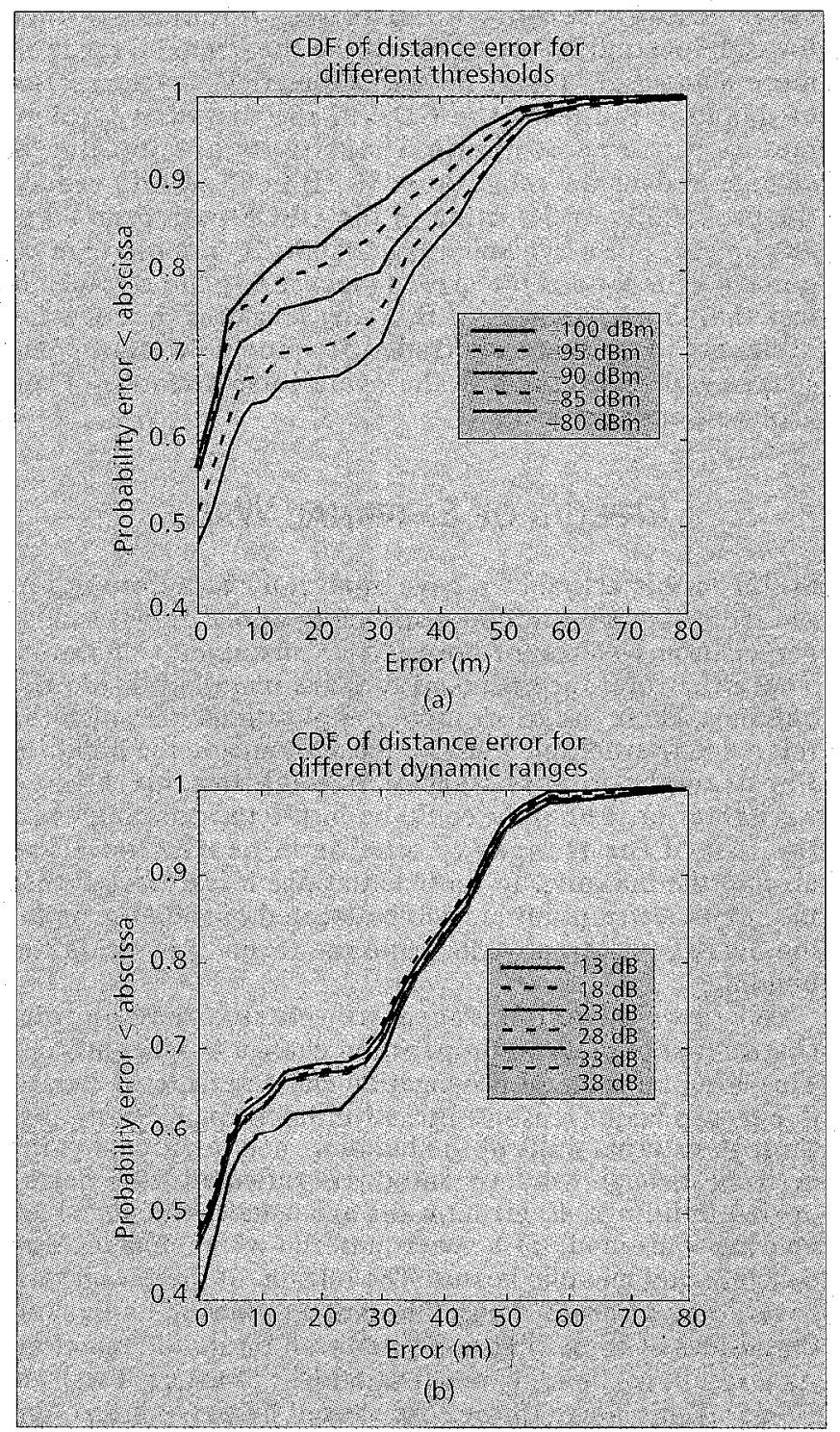

Figure 7. CDF of the error in predicted distance for the outdoor transmitter: a) as a function of different receiver sensitivies and $a$ dynamic range of $40 \mathrm{~dB}$; b) with a threshold of $-80 \mathrm{dBm}$ and different dynamic ranges. power for the DLOS path is $90 \mathrm{~dB}$ as compared to about 40 $\mathrm{dB}$ for the combined other paths and total received power. When compared to the results of Fig. 3 for indoor-to-indoor situations, the range of the power of the DLOS path in the outdoor-to-indoor case is significantly larger, while the range of total received power and the combined power in the other paths remains approximately the same. In many locations inside the building, the DLOS path has to pass through many walls, including a heavy outside wall, while other paths can enter the building through open doors and windows. In other words, we may have many locations in the building where we have reasonable power coming through the external windows and doors but the DLOS path is extremely weak.

Figure 7 shows the CDF of the error in predicted distance for a variety of sensitivities and dynamic ranges. When compared with the graphs for indoor-to-indoor applications shown in Fig. 5, the curves in Fig. 7 offer two classes of errors, one less than $\sim 10 \mathrm{~m}$ and the other more than about $30 \mathrm{~m}$. In the first group of profiles, when the DLOS path is not detected, the next detected path has arrived after penetration through the closest wall to the transmitter, so the arrival time is less than $30 \mathrm{~ns}(10 \mathrm{~m})$ delayed from the arrival time of the DLOS path. In the second group the falsely detected first path arrives through windows and doors from external reflections, so the overall path length is more than $90 \mathrm{~ns}(30 \mathrm{~m})$. Therefore, the error is either between the paths coming through penetration or those coming through windows and doors, and these two classes provide a distinct behavior in the error caused by the receiver.

\section{CONCLUSIONS}

Because of the frequency-selective multipath fading characteristics of the indoor radio channel, design of an accurate indoor geolocation system is a challenging task. To provide a foundation for quantitative performance evaluation of such systems a methodology for statistical modeling of this channel for geolocation applications is presented. To relate the performance of traditional GPS receivers to the more complex RAKE-type receivers, the multipath profiles in an indoor area were divided into three classes: DDP, NDDP, and UDP. In the DDP case both RAKE-type and traditional GPS receivers operate properly. In the NDDP case only RAKE receivers function accurately, and in the UDP case neither of the receivers is satisfactory. The statistics of the occurrence of the three classes of channel profiles in a building with harsh radio propagation characteristics were presented. The statistics of error in measuring the distance as a function of the sensitivity and dynamic range of the receiver as well as effects of outside walls were presented. As we move reference transmitters to the outside of the building, the statistics of the error in estimating the TOA of the DLOS will change significantly. The signal arriving from windows and doors through reflection from neighboring buildings will cause larger errors in prediction of the TOA of the DLOS path.

\section{ACKNOWLEDGMENTS}

The authors would like to express their appreciation to DARPA's SUO-SAS program for partial support of this project and approval for public release with unlimited distribution. We are thankful to Dr. Mark McHenry of DARPA for his comments and suggestions. We also thank Paul Creamer and Joseph Pisano, our collaborators at TASC, Reading, Massachusetts, and Dr. James W. Matthews and Ahmad 
Hatami, our associates at CWINS, WPI for fruitful discussions and a variety of help. We also appreciate Yan Xu's contribution in running the $R T$ program for the outdoor-to-indoor scenario.

\section{REFERENCES}

[1] K. Pahlavan and A. Leveqque, Wireless Information Networks, New York: Wiley, 1995.

[2] K. Pahlavan, A. Zahedi and P. Krishnamurthy, "Wideband Local Access: WLAN and WATM," IEEE Commun. Mag., Nov. 1997.

[3] K. Pahlavan, T. Probert and M. Chase, "Trends in Wireless Local Networks," IEEE Commun. Mag. Mar. 1995.

[4] MicroTRAX tracking and location system Web site, http://www. harris.com/microtrax.

[5] M. J. Meyer et al., "Wireless Enhanced 9-1-1 Service - Making It a Reality," Bell Labs Tech. J., pp. 188-202, Autumn 1996.

[6] "Report on the New Jersey Wireless Enhanced 911 System Trial," State of New Jersey, Dept. of Law and Public Safety, June 16, 1997.

[7] Trueposition Systems, http://www.trueposition.com.

[8] DARPA Tactical Technology Office: Situation Awareness System Open Review \#1 and \#2: htth://web-ext2.darpa.mil/tto/sas-docs.htm.

[9] P. K. Enge, "Global Positioning Systems: Signals, Measurements and Performance," Int'l. J. Wireless Info. Networks, vol. 1, no.2, Apr. 1994.

[10] I. A. Getting, "The Global Positioning System," IEEE Spectrum, Dec. 1995, pp. 36-47.

[11] E. D. Kaplan, ed., Understanding GPS: Principles and Applications, Boston: Artech House, 1996.

[12] S. J. Howard and K. Pahlavan, "Measurement and Analysis of the Indoor Radio Channel in the Frequency Domain," IEEE Trans. Instr. Meas., no. 39, 1990, pp. 751-55.

[13] T. Holt, K. Pahlavan and J. Lee, "A Graphical Indoor Radio Channel Simulator Using 2-D Ray Tracing," Proc. PIMRC '92, Oct. 1992, pp. 411-16.
[14] G. Yang and K. Pahlavan, "Sectored Antenna and DFE Modem for High Speed Indoor Radio Communications," IEEE Trans. Vehic. Tech., Nov. 1994.

[15] M. Hasan-Ali and K. Pahlavan, "Site-Specific Wideband Indoor Channel Modelling Using Ray Tracing Software," Elect. Lett., vol. 33, no. 23, Nov. 1997, pp. 1983-84.

[16] A. Falsafi, K. Pahlavan and G. Yang, "Transmission Techniques for Wireless LANs," IEEE JSAC, Apr. 1996.

\section{BIOGRAPHIES}

KaVeH PAHLAVAN [F] (kaveh@wpi.edu) is a professor of electrical and computer engineering and director of the Center for Wireless Information Network Studies (CWINS) at Worcester Polytechnic Institute (WPI), Massachusetts. His recent research has been focused on indoor radio propagation modeling and analysis of multiple access and transmission methods for wireless local networks. He is editor-in-chief and founder of the International Journal of Wireless information Networks, and has contributed to numerous technical papers and two patents. He has been program chair and organizer of IEEE conferences and workshops including PIMRC '98 this year. Additional infor-

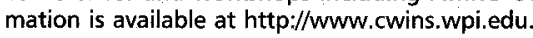

PRASHANT KRISHNAMURTHY (prashant@wpi.edu) is a research assistant at CWINS at WPI. His recent research has focused on statistical indoor radio propagation modeling for signal coverage and urban geolocation, developing tools for deployment of wireless LANs, and issues in mobile computing and wireless information networks.

JACQUES BENEAT (beneat@ece.wpi.edu) is a postdoctoral fellow at CWINS at WPI. He has helped in the organization of the Second IEEE Workshop on Wireless LANs held at WPI in 1996, and is currently assisting in the organization of the ninth IEEE Personal Indoor and Mobile Radio Communications conference, to be held in Boston in 1998. 